\section{Cellular Physiology} and Biochemistry Published online: December 07, 2015

Accepted: October 27, 2015

\title{
MicroRNA-138 Regulates Metastatic Potential of Bladder Cancer Through ZEB2
}

\author{
De-Kang Sun Jian-Ming Wang Peng Zhang Yong-Qiang Wang \\ Department of Urology, Yantai Yuhuangding Hospital, Yantai, China
}

\section{Key Words}

Bladder cancer $(\mathrm{BC}) \cdot \mathrm{ZEB} 2 \cdot \mathrm{MiR}-138 \cdot$ Cancer metastases

\begin{abstract}
Background/Aims: The cases of bladder cancer (BC) with poor prognosis largely result from the distal metastases of the primary tumor. Since microRNAs (miRNAs) play critical roles during cancer metastases, determination of the involved miRNAs in the regulation of the metastases of BC may provide novel therapeutic targets for BC treatment. Here, we aimed to study the role of miR-138 in regulation of BC cell invasion and metastases. Methods: We analyzed the levels of miR-138 and ZEB2, a key factor that regulates cancer cell invasion, in the $B C$ specimens from the patients. We also studied the correlation between miR-138 and ZEB2. We performed bioinformatics analyses on the binding of miR-138 to the 3'-UTR of ZEB2 $m R N A$, and verified the biological effects of this binding through promoter luciferase reporter assay. The effects of miR-138-modification on BC cell invasion were evaluated in a transwell cell invasion assay and a scratch would healing assay. Results: We found that the levels of miR-138 were significantly decreased and the levels of ZEB2 were significantly increased in $\mathrm{BC}$ specimens, compared to the paired normal bladder tissue. Metastatic BC appeared to contained lower levels of miR-138. Moreover, miR-138 and ZEB2 inversely correlated in BC specimens. Bioinformatics analyses showed that miR-138 targeted the 3'-UTR of ZEB2 mRNA to inhibit its translation. Furthermore, miR-138 overexpression inhibited ZEB2-mediated cell invasion and metastases, while miR-138 depletion increased ZEB2-mediated cell invasion and metastases in BC cells. Conclusion: Suppression of miR-138 in BC cells may promote ZEB2-mediated cancer invasion and metastases. Thus, miR-138 appears to be an intriguing therapeutic target to prevent metastases of BC.

\section{Introduction}

Many diagnosed bladder cancer (BC) were found to have reached advanced stages with distal metastases, which leads to poor prognosis. This feature of BCs largely results from the nature of $\mathrm{BC}$, being asymptomatic or non-specifically symptomatic in the early stages [1], and specifically emphasizes the importance of the comprehension of the molecular mechanisms underlying the regulation of BC metastases [2]. 


\section{Cellular Physiology Cell Physiol Biochem 2015;37:2366-2374 \begin{tabular}{l|l|l} 
and BOI: 10.1159/000438590 & $\begin{array}{l}\text { C } 2015 \text { The Author(s). Published by S. Karger AG, Basel } \\
\text { www.karger.com/cpb }\end{array}$
\end{tabular} \\ Sun et al.: MiR-138 Inhibits ZEB2-Mediated BC Metastases}

Epithelial-Mesenchymal Transition (EMT) is a critical biological event that triggers the modification of the cancer cell properties to allow cancer cell to adapt to a phenotype favoring invasiveness and metastases [3-6]. Among all EMT-associated proteins, the transcription factor ZEB1 and ZEB2 have been well-defined as essential EMT mediators [7]. ZEB1 and ZEB2 are critical for maintenance of an adapted mesenchymal cell phenotype, as well as for EMT induction [8]. Previous approaches have found that ZEB proteins possess zinc-finger clusters in all their $\mathrm{N}$-terminal, central and C-terminal regions to selectively bind to an E-box sequence CACCTG on the DNA of target genes, e.g. E-cadherin [8]. Loss of E-cadherin as well as activation of Vimentin is not only the trigger and dependent process, but also the manifestation for adaption of an epithelial cell to a mesenchymal phenotype.

MicroRNAs (miRNAs) are non-coding small RNAs, which are about 18-23 nucleotide long. MiRNAs regulate the protein translation of target genes, through base-pairing with the $3^{\prime}$-untranslated region (3'-UTR) of the mRNAs $[9,10]$. It is well-known that miRNAs regulate carcinogenesis [11-14]. Among all miRNAs, miR-138 has been extensively studied. Downregulation of miR-138 has been found to be associated with overexpression of human telomerase reverse transcriptase protein in human anaplastic thyroid carcinoma cell lines [15]. Moreover, miR-138 has been shown to reverse multidrug resistance of leukemia cells [16]. In addition, miR-138 was reported to suppress nasopharyngeal carcinoma growth and tumorigenesis by targeting the CCND1 oncogene [17]. And, miR-138 induces cell cycle arrest by targeting cyclin D3 in hepatocellular carcinoma [18]. All these studies suggest miR-138 as a potent tumor inhibitor. However, a role of miR-138 in BC has not been studied so far.

Here, we studied the $\mathrm{BC}$ specimens from the patients, and found that the levels of miR138 were significantly decreased and the levels of ZEB2, a critical factor that regulates cancer cell invasiveness, were significantly increased in $\mathrm{BC}$, compared to the paired normal bladder tissue. Metastatic BC appeared to contained lower levels of miR-138. Moreover, miR-138 and ZEB2 inversely correlated in BC specimens. Bioinformatics analyses showed that miR138 targeted the 3'-UTR of ZEB2 mRNA to inhibit its translation, which was confirmed in a luciferase-reporter assay. Further, miR-138 overexpression inhibited ZEB2-mediated cell invasion and metastases, while miR-138 depletion increased ZEB2-mediated cell invasion and metastases in $\mathrm{BC}$ cells.

\section{Materials and Methods}

Patient tissue specimens

A total of 30 resected specimens from BC patients were collected for this study (Table 1). BC specimens were compared with the paired normal bladder tissue (NT) from the same patient. All specimens had been histologically and clinically diagnosed at Yantai Yuhuangding Hospital from 2010 to 2014, independently by two experienced pathologists. For the use of these clinical materials for research purposes, prior patient's consents and approval from the Institutional Research Ethics Committee were obtained.

Culture of a human $B C$ cell line

A Human BC line T24 was purchased from American Type Culture Collection (ATCC, Rockville, MD, USA). T24 cells were cultured in RPMI 1640 medium supplemented with 15\% heat-inactivated fetal bovine serum (FBS; Sigma-Aldrich, St Louis, MO, USA), $100 \mathrm{U} / \mathrm{ml}$ penicillin and $100 \mu \mathrm{g} / \mathrm{ml}$ streptomycin (Invitrogen, Carlsbad, CA, USA) in a humidified atmosphere of $5 \% \mathrm{CO}_{2}$ at $37^{\circ} \mathrm{C}$.

Plasmids transfection

MiR-138-modulating and ZEB2-modulating plasmids were prepared using a backbone plasmid containing a GFP reporter under CMV promoter (pcDNA3.1-CMV-GFP, Clontech, Mountain View, CA, USA). The miR-138 mimic, or antisense, or control null, or short-hairpin interfering RNA for ZEB2 (shZEB2), or a control scrambled sequence was all purchased from Sigma-Aldrich, and digested with Xhol and BamHI and subcloned with a $2 \mathrm{~A}$ into a pcDNA3.1-CMV-GFP backbone. The small 2A peptide sequences, when cloned 


\section{Cellular Physiology Cell Physiol Biochem 2015;37:2366-2374 and Biochemistry Published online: December 07, 2015 le $\begin{aligned} & \text { (c) } 2015 \text { The Author(s). Published by S. Karger AG, Basel } \\ & \text { www.karger.com/cpb }\end{aligned}$ \\ Sun et al.: MiR-138 Inhibits ZEB2-Mediated BC Metastases}

Table 1. Clinical-pathological characteristics of BC specimens

\begin{tabular}{ll}
\hline & Patients (n; \%) \\
\hline BC tissue/ Adjacent non-tumor tissue & $30(100 \%) / 30(100 \%)$ \\
Age $(<50 / \geq 50$ years old) & $18(60 \%) / 12(40 \%)$ \\
Gender (male/female) & $19(63 \%) / 11(37 \%)$ \\
Tumor site (Bladder) & $30(100 \%)$ \\
Tumor grade (well or moderate/poor) & $15(50 \%) / 9(30 \%) / 6(20 \%)$ \\
Tumor size (T1/T2/T3/T4) & $10(33 \%) / 8(27 \%) / 9(30 \%) / 3(10 \%)$ \\
Lymph node metastasis (no/yes) & $12(40 \%) / 18(60 \%)$ \\
Distal metastasis (no/yes) & $18(60 \%) / 12(40 \%)$ \\
\hline
\end{tabular}

between genes, allow for efficient, stoichiometric production of discrete protein products within a single vector through a novel "cleavage" event within the $2 \mathrm{~A}$ peptide sequence. Sequencing was performed to confirm the correct orientation of the new plasmid. Transfection was performed with Lipofectamine 2000 reagent (Invitrogen), according to the instructions of the manufacturer. One day after transfection, the transfected cells were purified by flow cytometry based on their expression of GFP.

Western blot

The protein was extracted from the specimens, or from the cultured T24 cells, in RIPA lysis buffer (1\% NP40, 0.1\% SDS, $100 \mu \mathrm{g} / \mathrm{ml}$ phenylmethylsulfonyl fluoride, $0.5 \%$ sodium deoxycholate, in PBS) on ice. The supernatants were collected after centrifugation at $12000 \times \mathrm{g}$ at $4^{\circ} \mathrm{C}$ for $20 \mathrm{~min}$. Protein concentration was determined using a BCA protein assay kit (Bio-rad, China), and whole lysates were mixed with $4 \times$ SDS loading buffer (125 mmol/l Tris-HCl, 4\% SDS, 20\% glycerol, $100 \mathrm{mmol} / \mathrm{l}$ DTT, and 0.2\% bromophenol blue) at a ratio of $1: 3$. Samples were heated at $100^{\circ} \mathrm{C}$ for $5 \mathrm{~min}$ and were separated on SDS-polyacrylamide gels. The separated proteins were then transferred to a PVDF membrane. The membrane blots were first probed with a primary antibody. After incubation with horseradish peroxidase-conjugated second antibody, autoradiograms were prepared using the enhanced chemiluminescent system to visualize the protein antigen. The signals were recorded using X-ray film. Primary antibodies were rabbit anti-ZEB2, anti-E-cadherin, anti-Vimentin and anti- $\alpha$-tubulin (Cell Signaling, San Jose, CA, USA). Secondary antibody is HRP-conjugated anti-rabbit (Jackson ImmunoResearch Labs, West Grove, PA, USA). Blotting images were representatives from 5 repeats. $\alpha$-tubulin was used as a protein loading control.

\section{RT-qPCR}

Total RNA was extracted from resected specimens or from cultured cells with miRNeasy mini kit (Qiagen, Hilden, Germany) for cDNA synthesis. Complementary DNA (cDNA) was randomly primed from $2 \mu \mathrm{g}$ of total RNA using the Omniscript reverse transcription kit (Qiagen). Quantitative PCR (RT-qPCR) were performed in duplicates with QuantiTect SYBR Green PCR Kit (Qiagen). All primers were purchased from Qiagen. Data were collected and analyzed using $2^{-\Delta \Delta \mathrm{Ct}}$ method for quantification of the relative mRNA expression levels. Values of genes were first normalized against $\alpha$-tubulin, and then compared to controls.

\section{Luciferase-reporter assay}

Luciferase-reporters were successfully constructed using molecular cloning technology. Target plasmids for 3'-UTR of ZEB2 mRNA clone or 3'-UTR of ZEB2 mRNA with a mutant at miR-138 binding sites wre purchased from Creative Biogene (Shirley, NY, USA). MiR-138-modified T24 cells were seeded in 24-well plates for 24 hours, after which they were transfected with $1 \mu \mathrm{g}$ of Luciferase-reporter plasmids per well. Luciferase activities were measured using the dual-luciferase reporter gene assay kit (Promega, Beijing, China), according to the manufacturer's instructions. The normalized control was null-transfected T24 cells with 3'-UTR of ZEB2 mRNA (wild type).

Transwell cell invasion assay

Cells $\left(10^{4}\right)$ were plated into the top side of polycarbonate transwell filter coated with Matrigel in the upper chamber of the BioCoatTM Invasion Chambers (Becton-Dickinson Biosciences, Bedford, MA, USA) and incubated at $37^{\circ} \mathrm{C}$ for 22 hours. The cells inside the upper chamber with cotton swabs were then removed. 


\section{Cellular Physiology Cell Physiol Biochem 2015;37:2366-2374 \begin{tabular}{ll|l} 
DOI: 10.1159/000438590 & $\begin{array}{l}\text { O 2015 The Author(s). Published by S. Karger AG, Basel } \\
\text { www.karger.com/cph }\end{array}$
\end{tabular} \\ Sun et al.: MiR-138 Inhibits ZEB2-Mediated BC Metastases}

Migratory and invasive cells on the lower membrane surface were fixed, stained with hematoxylin, and counted for 10 random $100 X$ fields per well. Cell counts are expressed as the mean number of cells per field of view. Five independent experiments were performed and the data are presented as mean \pm standard deviation (SD).

\section{Scratch wound healing assay}

Scratch wound healing assay was performed as has been described previously [19]. Cells were seeded in 24-well plates at a density of $10^{4}$ cells/well in complete media and cultured to confluence. The cell monolayer was serum starved overnight in media prior to initiating of the experiment. Confluent cell monolayer were then scraped with a yellow pipette tip to generate scratch wounds and washed twice with media to remove cell debris. Cells were incubated at $37^{\circ} \mathrm{C}$ for 24 hours. Time lapse images were captured after 12 hours. Images were captured from 5 randomly selected fields in each sample, and the migration areas are determined by subtracting the wound area at the indicated time periods from the initial wound area, using by NIH ImageJ software (Bethesda, MA, USA), as has been previously described [20].

\section{Statistical analysis}

All statistical analyses were carried out using the SPSS 17.0 statistical software package. Bivariate correlations were calculated by Spearman's Rank Correlation Coefficients. All values are depicted as mean \pm $\mathrm{SD}$ and are considered significant if $\mathrm{p}<0.05$. All data were statistically analyzed using one-way ANOVA with a Bonferroni correction, followed by Fisher's Exact Test for comparison of two groups.

\section{Results}

Decreased miR-138 correlates with increased ZEB2 in $B C$

In the BC samples (Table 1), we detected significantly higher levels of ZEB2, compared to paired normal bladder tissue (NT; Fig. 1A). Moreover, we detected significantly lower levels of miR-138 in BC, compared to NT (Fig. 1B). In order to examine the relationship between miR-138 and ZEB2 in BC, we performed the correlation test using the $30 \mathrm{BC}$ specimens. A strong inverse correlation was detected between miR-138 and ZEB2 (Fig. 1C, $\gamma=-0.74$, $\mathrm{p}<0.0001$ ), suggesting the presence of a relationship between miR-138 and ZEB2 in BC.

Fig. 1. Decreased miR138 correlates with increased ZEB2 in BC specimens. RT-qPCR on miR-138 and Western blot for ZEB2 were performed on paired $\mathrm{BC}$ and the adjacent normal Bladder tissues (NT) from 30 patients. (A-B) $B C$ contained increased ZEB2 levels by $6.5 \pm 1.0$ fold (A), and decreased miR-138 levels by $70 \pm 15 \%$ (B), compared to NT. (C) A correlation test between ZEB2 and miR-138 $(\gamma=-0.74$, $\mathrm{p}<0.0001$ ). (D) The miR-138 levels in $\mathrm{BC}$
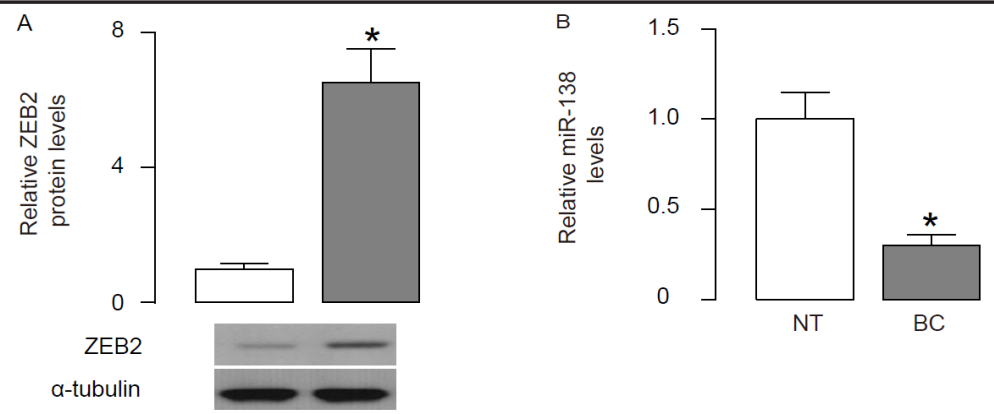

NT BC
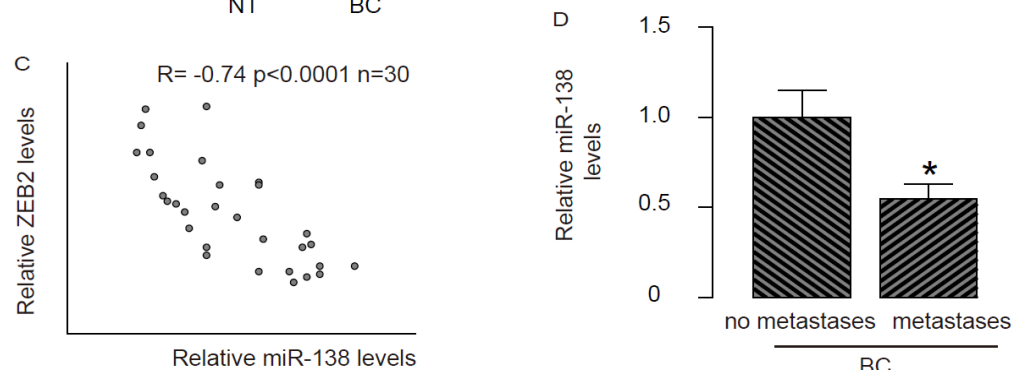
with/without metastases. BC with metastases had a lower levels of miR-138 by $45 \pm 6.5 \% * p<0.05$. $\mathrm{N}=30$. All values are depicted as mean $\pm \mathrm{SD}$. 


\section{Cellular Physiology Cell Physiol Biochem 2015;37:2366-2374

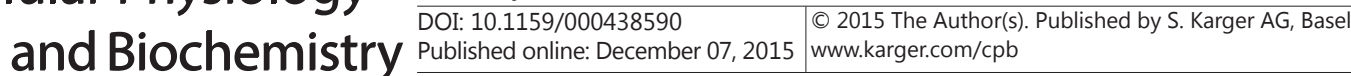 \\ Sun et al.: MiR-138 Inhibits ZEB2-Mediated BC Metastases}

Fig. 2. MiR-138 targets 3'-UTR of ZEB2 mRNA to inhibit its protein translation. (A) Bioinformatics analyses of binding of miR-138 to the 3'-UTR of ZEB2 mRNA. (B-C) We either overexpressed miR-138, or inhibited miR-138 in T24 cells by transfecting the cells with a miR-138-expressing plasmid (T24-miR-138), or with a plasmid carrying miR-138 antisense (T24as-miR-138). The T24 cells were also transfected with a null plasmid as a control (T24-null). (B) RT-qPCR for miR-138 in miR-138 modified T24 cells. (C) MiR-138-modified T24 cells were then transfected with 1 $\mu$ g of ZEB2 3 '-UTR luciferase-reporter plasmid. Moreover, null-transfected T24 cells were also transfected with $1 \mu \mathrm{g}$ plasmids carrying luciferase reporter for 3'-UTR of ZEB2 mRNA with one mutate at the miR-138 binding site (mut). The luciferase activities were quantified. $* \mathrm{p}<0.05 . \mathrm{N}=5$.

Since ZEB2 is an EMT-associated protein to promote cancer cell metastases, we separated the $30 \mathrm{BC}$ specimens into 2 groups based on the distal cancer metastases. We found that $\mathrm{BC}$ that had distal metastases contained significantly lower levels of miR-138 (Fig. 1D).

\section{MiR-138 targets 3'-UTR of ZEB2 mRNA to inhibit its protein translation}

Since our data suggest a relationship between miR-138 and ZEB2 in BC cells, we checked whether miR-138 may regulate ZEB2 protein translation. Using bioinformatics analyses, we detected miR-138 binding sites on 3'-UTR (from 766th base site to 772th base site) of the ZEB2 mRNA (Fig. 2A). In order to examine whether the binding of miR-138 to ZEB2 mRNA may affect ZEB2 protein translation in BC cells, we either overexpressed miR-138, or inhibited miR-138 in a human BC cell line T24, through transfecting the cells with a miR-138expressing plasmid (T24-miR-138), or with a plasmid carrying miR-138 antisense (T24-asmiR-138). The T24 cells were also transfected with a null plasmid as a control (T24-Nul). Co-expression of a GFP reporter in these plasmids allows purification of transfected cells by flow cytometry. The overexpression or inhibition of miR-138 in these cells was confirmed by RT-qPCR (Fig. 2B). MiR-138-modified T24 cells were then transfected with $1 \mu \mathrm{g}$ plasmids carrying luciferase reporter for 3'-UTR of ZEB2 mRNA. Moreover, null-transfected T24 cells were also transfected with $1 \mu \mathrm{g}$ plasmids carrying luciferase reporter for 3'-UTR of ZEB2 mRNA with one mutate at the miR-138 binding site (mut). The luciferase activities were quantified in these cells, suggesting that miR-138 specifically targets 3'-UTR of ZEB2 mRNA to inhibit its translation (Fig. 2C).

MiR-138 reduces ZEB2 protein in BC cells

Next, we analyzed the modification of miR-138 levels in T24 cells on ZEB2 and other EMT-associated proteins Vimentin and E-cadherin. In order to confirm that the effects of miR-138 on Vimentin and E-cadherin are exerted through modulation of ZEB2, we further 
Fig. 3. MiR-138 reduces ZEB2 protein in BC cells. (A) RT-qPCR for ZEB2 in miR-138 (plus ZEB2)-modified cells. (B-D) Western blot for ZEB2 (B), Vimentin (C) and E-cadherin (D) in miR-138 (plus ZEB2)-modified T24 cells. ${ }^{*} \mathrm{p}<0.05$. NS: non-significant. $\mathrm{N}=5$.

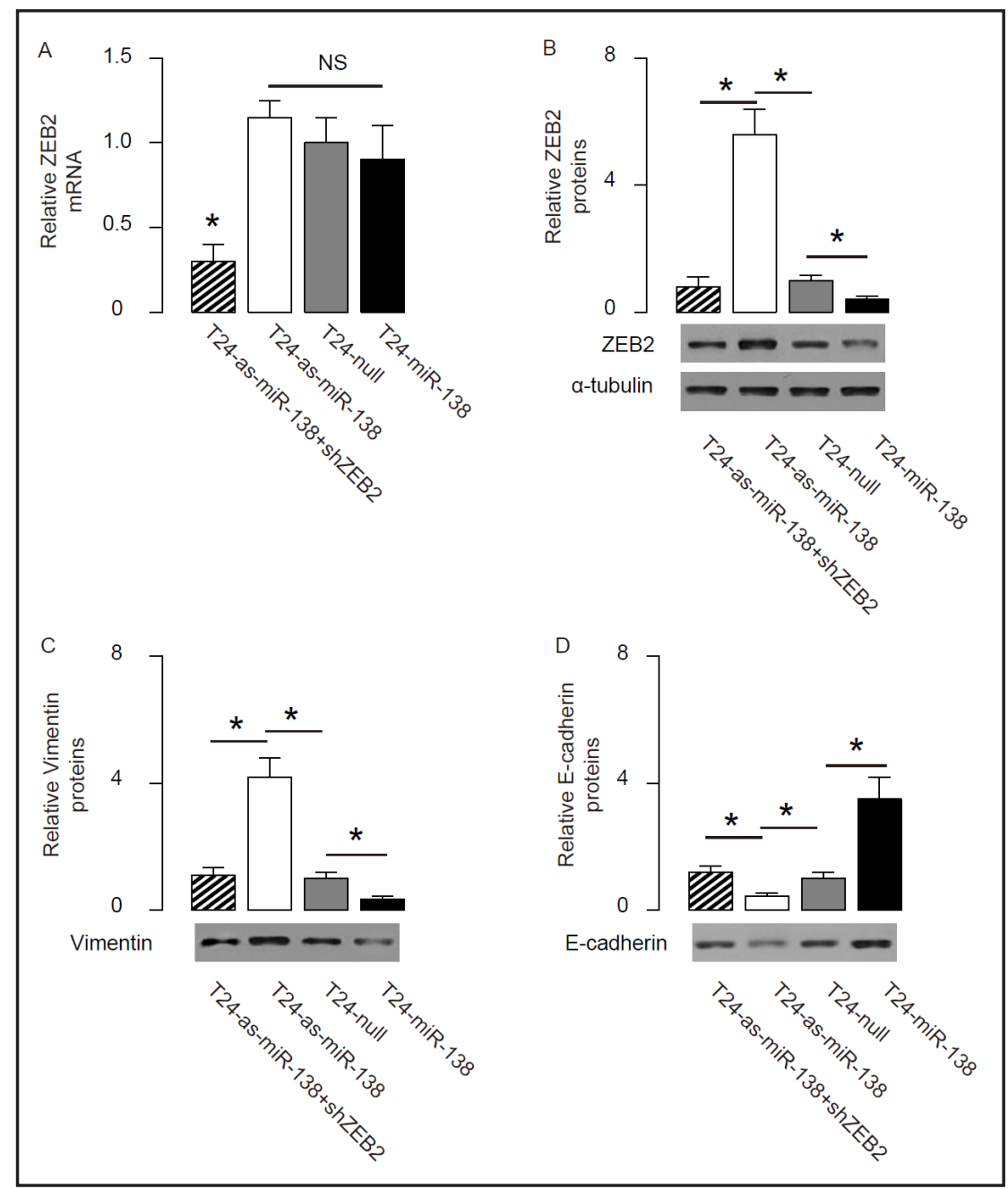

knocked down ZEB2 by shRNA in T24 cells that expressed antisense of miR-138 (T24as-miR-138-shZEB2), using a scrambled sequence as a control transfection. We found that alteration of miR-138 in T24 cells did not change ZEB2 mRNA (Fig. 3A). However, overexpression of miR-138 significantly decreased ZEB2 protein (Fig. 3B) and Vimentin (Fig. 3C), and significantly increased E-cadherin (Fig. 3D). On the other hand, inhibition of miR138 significantly increased ZEB2 protein (Fig. 3B) and Vimentin (Fig. 3C), and significantly decreased E-cadherin (Fig. 3D). Suppression of ZEB2 abolished the effects of as-miR-138 expression on Vimentin and E-cadherin (Fig. 3C-D). Together, these data suggest that MiR138 inhibits ZEB2 protein translation in T24 cells.

\section{MiR-138 suppresses BC cell invasion and migration}

We found that overexpression of miR-138 resulted in decreases in cell invasion of T24 cells in a transwell cell invasion assay, shown by quantification (Fig. 4A), and by representative images (Fig. 4B). Similarly, depletion of miR-138 resulted in increases in cell invasion of T24 cells, shown by quantification (Fig. 4A), and by representative images (Fig. 4B). Suppression of ZEB2 abolished the effects of as-miR-138 expression on cell invasion (Fig. 4A-B). Using scratch wound healing assay, we found that overexpression of miR-138 resulted in decreases in cell migration in vitro, shown by quantification (Fig. 5A), and by representative images (Fig. 5B). Similarly, depletion of miR-138 resulted in increases in cell migration in vitro, shown by quantification (Fig. 5A), and by representative images (Fig. 5B). Suppression of ZEB2 abolished the effects of as-miR-138 expression on cell migration (Fig. 
Fig. 4. MiR-138 suppresses $\mathrm{BC}$ cell invasion in a transwell cell invasion assay. (AB) Cell invasiveness of miR138 (plus ZEB2)-modified BC cells in a transwell cell invasion assay, shown by quantification (A), and by representative images (B). $* \mathrm{p}<0.05 . \mathrm{N}=5$.

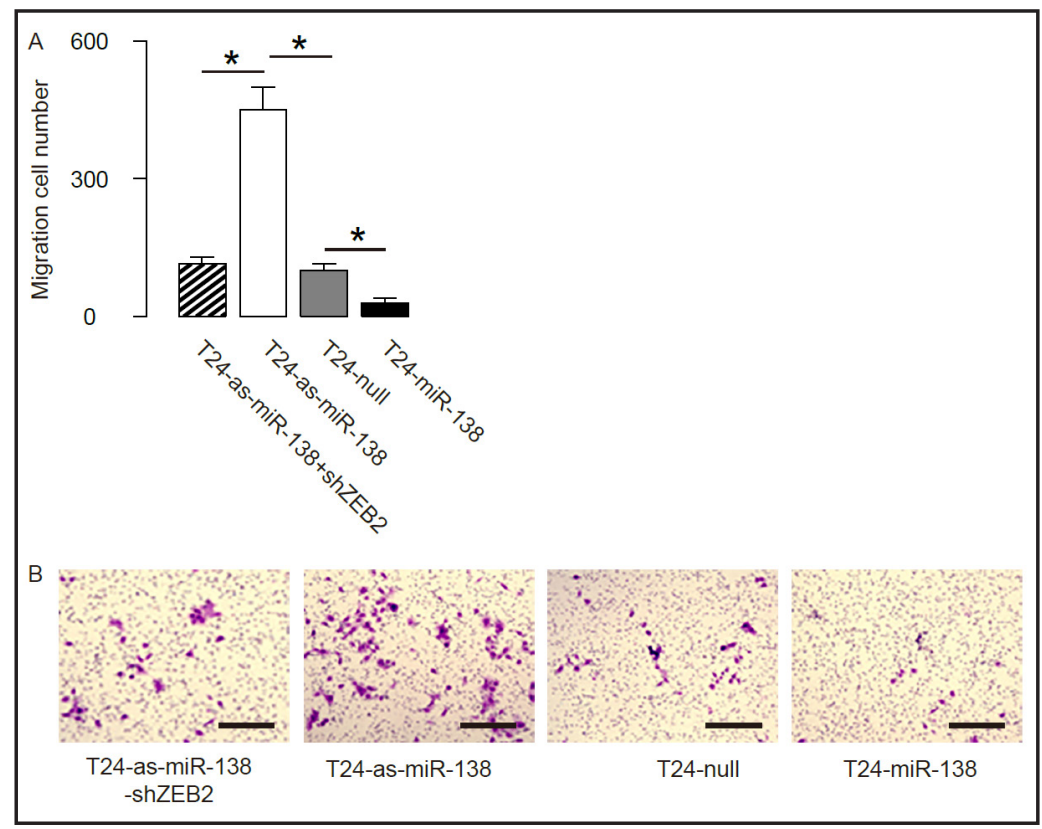

Fig. 5. MiR-138 suppresses $\mathrm{BC}$ cell migration in a scratch wound healing assay. (A-B) Cell invasiveness of miR-138 (plus ZEB2)-modified BC cells in a scratch wound healing assay, shown by quantification (A), and by representative images (B). $* \mathrm{p}<0.05 . \mathrm{N}=5$.

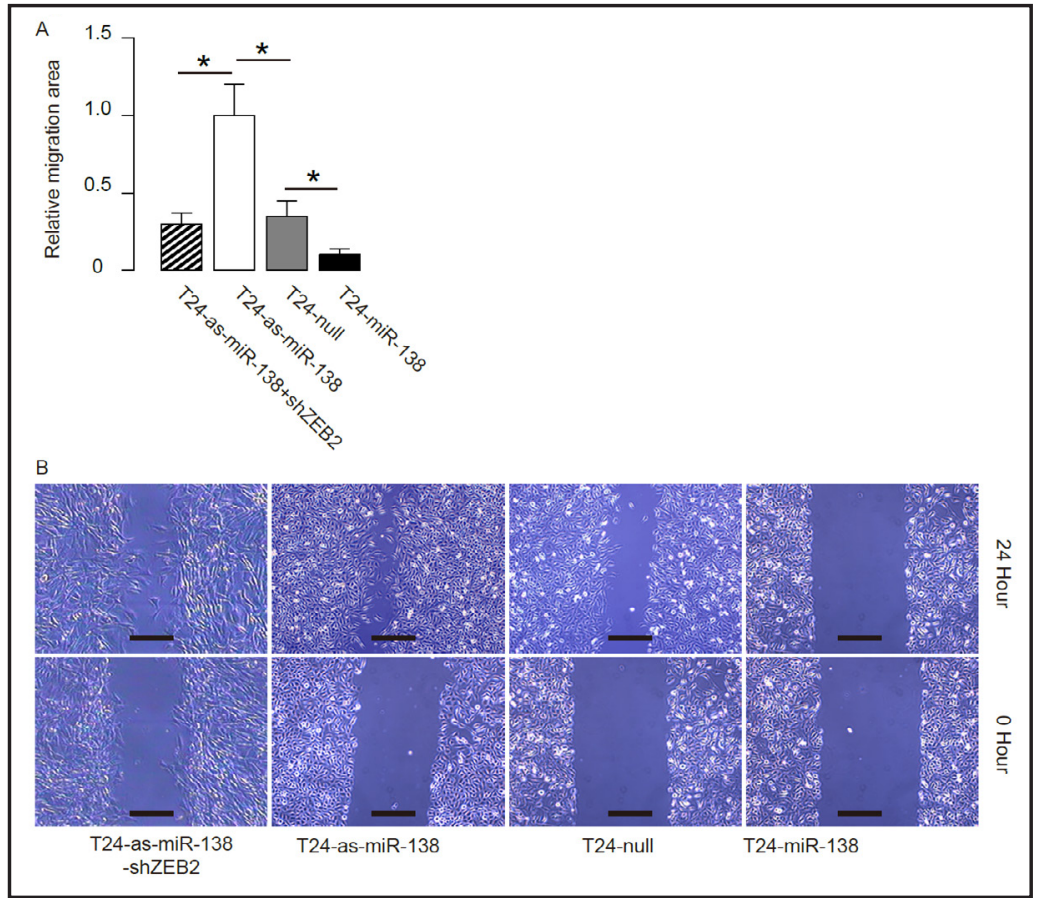

Fig. 6. A schematic model. MiR-138 inhibits BC cell invasion and migration through ZEB2 suppression.

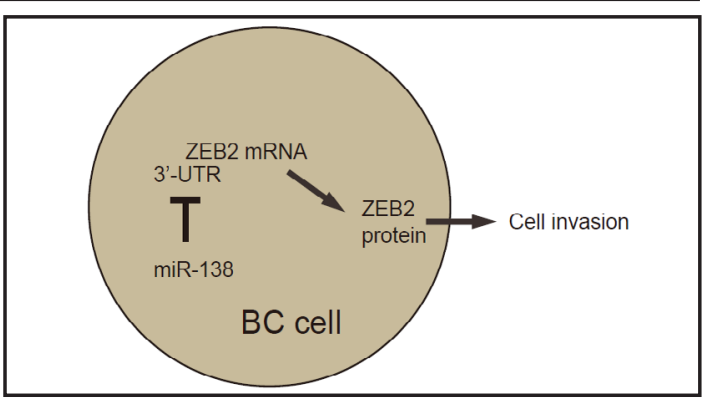

5A-B). Together, these data suggest that miR-138 inhibits BC cell invasion and migration through ZEB2 suppression (Fig. 6). 


\section{Cellular Physiology Cell Physiol Biochem 2015;37:2366-2374 and Biochemistry Published online: December 07, 2015 le $\begin{aligned} & \text { DOI: } 2015 \text { The Author(s). Published by S. Karger AG, Basel } \\ & \text { www.karger.com/cpb }\end{aligned}$ \\ Sun et al.: MiR-138 Inhibits ZEB2-Mediated BC Metastases}

\section{Discussion}

Various miRNAs play important roles in the invasion and metastases of malignant tumor cells. Specifically, miR-138 has been defined as a tumor suppressor in many types of cancer. For example, downregulation of miR-138 has been found to be associated with overexpression of human telomerase reverse transcriptase protein in human anaplastic thyroid carcinoma cell lines [15]. Moreover, miR-138 has been shown to reverse multidrug resistance of leukemia cells [16]. In addition, miR-138 was reported to suppress nasopharyngeal carcinoma growth and tumorigenesis by targeting the CCND1 oncogene [17]. And, miR-138 induces cell cycle arrest by targeting cyclin D3 in hepatocellular carcinoma [18]. BCR-ABL/GATA1/miR-138 mini circuitry has been reported to contribute to the leukemogenesis of chronic myeloid leukemia [21]. Down-regulation of miR-138 promotes colorectal cancer metastasis via directly targeting TWIST2 [22]. In lung cancer, miR-138 was found to inhibit tumor growth through repression of EZH2 [23], and to reverse gefitinib resistance via negatively regulating G protein-coupled receptor 124 [24]. Specifically, a recent report has shown that miR-138 induced marked reduction in vimentin expression and enhanced E-cadherin expression, characteristics of EMT, in squamous cell carcinoma cell lines. The authors further identified a number of miR-138 target genes that are associated with EMT, including VIM, ZEB2 and EZH2 (enhancer of zeste homologue 2) [25]. These previous studies encouraged us to study a role of miR-138 in the regulation of EMT-mediated BC cell invasion and metastases.

In the current study, we first reported the involvement of miR-138 as a tumor suppressor in BC. By sequence matching, we found a number of candidate miRNAs that target ZEB2, including miR-138, miR-141, miR-142, miR-200, miR-208, miR-218, miR-182, miR-4262, etc.

Among all these miRNAs, we specifically detected a significant decrease in miR-138 in $\mathrm{BC}$ specimens, compared to NT. Hence, we hypothesize that miR-138 may target and regulate ZEB2 in BC cells. Correlation test supported our hypothesis, showing that the levels of miR138 in BC tissues were inversely correlated with the levels of ZEB2. Moreover, metastatic BC contained even lower levels of miR-138.

Using in vitro assay, we further demonstrate that alteration in miR-138 levels does not affectZEB2 mRNA, but regulated ZEB2 protein level. Using promoter luciferase assay, we found that miR-138 inhibited ZEB2 through translation suppression. Moreover, miR-138-induced ZEB2 in BC cells directly regulated cell invasion and migration, checked independently in transwell cell invasion assay and scratch wound healing assay. Most interestingly, we found that miR-138 nearly exclusively targeted and inhibited ZEB2 among most important EMTassociated proteins, e.g. miR-138 did not target ZEB1, Snail, or Slug, in T24 cells. These data suggest that miR-138/ZEB2 regulatory axis may play a critical role in regulation of BC cell invasion and migration.

To summarize, we propose a model that miR-138 suppresses BC metastases through ZEB2 inhibition. Downregulation of miR-138 appears to directly contribute to the distal metastases of primary BC and subsequently poor prognosis. Thus, our study highlights miR138 as a promising novel target for treating $\mathrm{BC}$ and preventing $\mathrm{BC}$ metastases.

\section{Disclosure Statement}

The authors have declared that no competing interests exist.

\section{References}

1 Jin Y, Lu J, Wen J, Shen Y, Wen X: Regulation of growth of human bladder cancer by mir-192. Tumour Biol 2015;36:3791-3797.

2 Tan MY, Mu XY, Liu B, Wang Y, Bao ED, Qiu JX, Fan Y: Sumo-specific protease 2 suppresses cell migration and invasion through inhibiting the expression of mmp13 in bladder cancer cells. Cell Physiol Biochem 2013;32:542-548. 


\section{Cellular Physiology Cell Physiol Biochem 2015;37:2366-2374 \begin{tabular}{l|l|l} 
DOI: 10.1159/000438590 & (c) 2015 The Author(s). Published by S. Karger AG, Basel \\
www.karger.com/cpb
\end{tabular} \\ Sun et al.: MiR-138 Inhibits ZEB2-Mediated BC Metastases}

3 Hu Q, Tong S, Zhao X, Ding W, Gou Y, Xu K, Sun C, Xia G: Periostin mediates tgf-beta-induced epithelial mesenchymal transition in prostate cancer cells. Cell Physiol Biochem 2015;36:799-809.

4 Sa Y, Li C, Li H, Guo H: Timp-1 induces alpha-smooth muscle actin in fibroblasts to promote urethral scar formation. Cell Physiol Biochem 2015;35:2233-2243.

5 Lan A, Qi Y, Du J: Akt2 mediates tgf-beta1-induced epithelial to mesenchymal transition by deactivating gsk3beta/snail signaling pathway in renal tubular epithelial cells. Cell Physiol Biochem 2014;34:368-382.

6 Teng Y, Zhao L, Zhang Y, Chen W, Li X: Id-1, a protein repressed by mir-29b, facilitates the tgfbeta1-induced epithelial-mesenchymal transition in human ovarian cancer cells. Cell Physiol Biochem 2014;33:717-730.

7 Guo Y, Lang X, Lu Z, Wang J, Li T, Liao Y, Jia C, Zhao W, Fang H: Mir-10b directly targets zeb1 and pik3ca to curb adenomyotic epithelial cell invasiveness via upregulation of e-cadherin and inhibition of akt phosphorylation. Cell Physiol Biochem 2015;35:2169-2180.

8 Schmalhofer 0, Brabletz S, Brabletz T: E-cadherin, beta-catenin, and zeb1 in malignant progression of cancer. Cancer Metastasis Rev 2009;28:151-166.

9 Di Leva G, Croce CM: Mirna profiling of cancer. Curr Opin Genet Dev 2013;23:3-11.

10 Pereira DM, Rodrigues PM, Borralho PM, Rodrigues CM: Delivering the promise of mirna cancer therapeutics. Drug Discov Today 2013;18:282-289.

11 Mei Q, Li F, Quan H, Liu Y, Xu H: Busulfan inhibits growth of human osteosarcoma through mir-200 family micrornas in vitro and in vivo. Cancer Sci 2014;105:755-762.

12 Wang F, Xiao W, Sun J, Han D, Zhu Y: Mirna-181c inhibits egfr-signaling-dependent mmp9 activation via suppressing akt phosphorylation in glioblastoma. Tumour Biol 2014;35:8653-8658.

13 Liu G, Jiang C, Li D, Wang R, Wang W: Mirna-34a inhibits egfr-signaling-dependent mmp7 activation in gastric cancer. Tumour Biol 2014;35:9801-9806.

14 Wu N, Zhang C, Bai C, Han YP, Li Q: Mir-4782-3p inhibited non-small cell lung cancer growth via usp14. Cell Physiol Biochem 2014;33:457-467.

15 Mitomo S, Maesawa C, Ogasawara S, Iwaya T, Shibazaki M, Yashima-Abo A, Kotani K, Oikawa H, Sakurai E, Izutsu N, Kato K, Komatsu H, Ikeda K, Wakabayashi G, Masuda T: Downregulation of mir-138 is associated with overexpression of human telomerase reverse transcriptase protein in human anaplastic thyroid carcinoma cell lines. Cancer Sci 2008;99:280-286.

16 Zhao X, Yang L, Hu J, Ruan J: Mir-138 might reverse multidrug resistance of leukemia cells. Leuk Res 2010;34:1078-1082.

17 Liu X, Lv XB, Wang XP, Sang Y, Xu S, Hu K, Wu M, Liang Y, Liu P, Tang J, Lu WH, Feng QS, Chen LZ, Qian CN, Bei JX, Kang T, Zeng YX: Mir-138 suppressed nasopharyngeal carcinoma growth and tumorigenesis by targeting the ccnd1 oncogene. Cell Cycle 2012;11:2495-2506.

18 Wang W, Zhao LJ, Tan YX, Ren H, Qi ZT: Mir-138 induces cell cycle arrest by targeting cyclin d3 in hepatocellular carcinoma. Carcinogenesis 2012;33:1113-1120.

19 Liang CC, Park AY, Guan JL: In vitro scratch assay: A convenient and inexpensive method for analysis of cell migration in vitro. Nat Protoc 2007;2:329-333.

20 Simoes AE, Pereira DM, Gomes SE, Brito H, Carvalho T, French A, Castro RE, Steer CJ, Thibodeau SN, Rodrigues CM, Borralho PM: Aberrant mek5/erk5 signalling contributes to human colon cancer progression via nf-kappab activation. Cell Death Dis 2015;6:e1718.

21 Xu C, Fu H, Gao L, Wang L, Wang W, Li J, Li Y, Dou L, Gao X, Luo X, Jing Y, Chim CS, Zheng X, Yu L: Bcr-abl/ gata1/mir-138 mini circuitry contributes to the leukemogenesis of chronic myeloid leukemia. Oncogene 2014;33:44-54.

22 Long L, Huang G, Zhu H, Guo Y, Liu Y, Huo J: Down-regulation of mir-138 promotes colorectal cancer metastasis via directly targeting twist2. J Transl Med 2013;11:275.

23 Zhang H, Zhang H, Zhao M, Lv Z, Zhang X, Qin X, Wang H, Wang S, Su J, Lv X, Liu H, Du W, Zhou W, Chen X, Fei K: Mir-138 inhibits tumor growth through repression of ezh2 in non-small cell lung cancer. Cell Physiol Biochem 2013;31:56-65.

24 Gao Y, Fan X, Li W, Ping W, Deng Y, Fu X: Mir-138-5p reverses gefitinib resistance in non-small cell lung cancer cells via negatively regulating g protein-coupled receptor 124. Biochem Biophys Res Commun 2014;446:179-186.

25 Liu X, Wang C, Chen Z, Jin Y, Wang Y, Kolokythas A, Dai Y, Zhou X: Microrna-138 suppresses epithelialmesenchymal transition in squamous cell carcinoma cell lines. Biochem J 2011;440:23-31. 